\title{
CHEST PHYSIOTHERAPY AND REBOUND EXERCISE FOR SPUTUM IN PATIENTS CO-INFECTED WITH TB AND HIV
}

\begin{abstract}
Patients co-infected with human immunodeficiency virus and tuberculosis find difficulty producing sputum for microscopy culture and sensitivity test. Chest physiotherapy induces sputum but ease of bacilli transmission and nosocomial infections necessitate minimal patient interaction. Rebound exercise has potential to facilitate sputum induction but its suitability and effect with this population is uncertain. This randomized controlled pre-and post-test study used 58 patients assigned equally to chest physiotherapy or a combination with rebound exercise for 30 minutes over three days to compare cardiopulmonary parameters and sputum volumes.

Heart, respiratory, blood pressure, oxygen saturation rates and sputum volume collected in a calibrated vial were monitored and recorded pre-and post-interventions. The combination of chest physiotherapy with rebound exercise produced significantly more sputum, higher respiratory and blood pressure rates (pre vs post; $p<0.05$ ). Acid-fast bacilli sensitivity was also higher following the combination compared to chest physiotherapy (54\% vs.38\%). Heart and oxygen saturation rates were not significant for both groups (pre-vs post; $p>0.05$ ). Rebound exercise when combined with chest physiotherapy could be a useful adjuvant to produce more sputum, higher sensitivity yield for acid-fast bacilli and reduce direct patient interaction but should be used with caution due to increased respiratory and blood pressure rates.
\end{abstract}

KEYWORDS: REBOUND EXERCISE, SIGNIFICANT, ADJUVANT, INDUCTION

\section{INTRODUCTION}

The province of KwaZulu-Natal has a population of approximately 9.5 million with the second highest tuberculosis (TB) incidence in South Africa (Naidoo and Jinabhai 2006). In sub-Saharan Africa up to $60 \%$ of TB patients are co-infected with the human immunodeficiency virus (HIV) which suppresses the immune system and makes the individual susceptible to opportunistic infections like pulmonary tuberculosis (PTB) which is spread by airborne droplets. Microbiological diagnosis using

\section{Corresponding Author:}

Dr Sonill S Maharaj

Head: Department of Physiotherapy

School of Health Sciences

University of KwaZulu-Natal

Private Bag X54001

Durban 4000 South Africa

Email:maharajss@ukzn.ac.za sputum has been the standard for PTB detection with patients referred to physiotherapists for chest physiotherapy and induced sputum specimens. Chest physiotherapy drains bronchial segments using gravity, manual percussions, vibrations, shaking of the chest wall, and diaphragmatic breathing to move secretions in a cephalad direction (Elkins et al. 2005). Assisted by vigorous coughing, secretions are cleared, resulting in improved ventilation and reducing proteolytic destruction of airways and the risk of secondary chest infections (Pryor and Webber 1999). However, a review of chest physiotherapy reported that this technique may only be effective in patients who produce more than 20-30mL of sputum (McCool and Rosen 2006). It was also reported that percussions and vibrations of the chest showed an increased expiratory flow rate, but it is not certain whether these techniques aid the removal of secretions (Mac Lean et al. 1989) suggesting that chest physiotherapy be discontinued for sputum induction (Elkins et al. 2005). An alternate method to facilitate sputum removal in children presenting with cystic fibrosis was regular aerobic exercises which could potentially replace conventional chest physiotherapy (Zach et al. 1982). However a period of bicycle ergometer exercise in adults having cystic fibrosis showed no significant increase in sputum extraction, and was the least effective when compared to postural drainage and positive expiratory pressure, indicating that exercises should be used to aid rather than substitute for chest physiotherapy (Olseni and Wollmer 1990; Salh et al. 1989). Generally, exercises are known to improve immune function and quality of life of HIV-infected individuals by improving lung function, breathing, ventilation, physical and emotional status, and delaying AIDSrelated complications (Stringer 1999). 
However HIV-infected individuals may have difficulty engaging in exercises due to lowered capacity to utilise oxygen and perform work because of the sideeffects of medication associated with HAART, such as zidovudine and didanosine, which have negative and varying effects on exercise tolerance and motivation (Keyser et al. 2000; Smit et al. 2006). It is also uncertain if the various treadmill protocols would be suitable for respiratory compromised patients with TB and HIV co-infection. Rebound exercise performed on a mini-trampoline with an elastic surface, springs and gravity-assisted vertical movements, is a relatively new type of exercise recommended inter alia to improve cardiorespiratory fitness (Carter 1988). A study comparing rebound exercise and cycle ergometry in HIV children, showed that rebound exercise produced a larger volume of sputum in a shorter period of time and could be used to enhance sputum extraction (Maharaj and Jeena 2008). In addition, mobilisation in standing, which is possible with gentle bouncing on the trampoline, improves functional residual capacity, oxygen saturation, partial pressure oxygen, and reduces partial pressure carbon dioxide with short-term improvements in tidal volume, inspiratory flow rate and minute volume (Zafiropoulos et al. 2004). It is also noted that any exercise used for respiratory compromised patients should be at low to moderate intensity, otherwise the individual will fatigue. Rebound exercise can vary in intensity according to the age and fitness of the individual, with feet either in constant contact as in a bouncing movement, or raised above the elastic surface depending on jump height assisted by gravity, springs and a handlebar for support. Healthcare workers in South Africa are at the forefront in the treatment of TB and HIV, with the incidence and risk of developing TB being high in KwaZuluNatal especially among paramedical staff, such as laboratory technicians, radiographers and physiotherapists (Naidoo and Jinabhai 2006). Owing to the large number of patients referred to physiotherapists, limited resources in public hospitals and sputum collected in small confined areas, it is possible that although the necessary precautions are taken against cross infection, the interaction with TB-infected patients during sputum induction could be a potential risk. In addition, HIV associated lung disease mimics the clinical and radiological picture of PTB (Zar et al. 2000) and lack of cavitations in TB and HIV co-infection result in lower numbers of bacilli in sputum, making identification of acid-fast bacilli (AFB) difficult, and delaying PTB diagnosis (Zumla et al. 2000). This therefore warrants alternate ways to minimise direct contact with TB-infected patients and improving the yield of AFB when evaluating sputum (Pinto and Bammann 2007). Chest physiotherapy is traditionally used to induce sputum, and moderate intensity rebound exercise, which is simple and requires less patient interaction, could be a possible adjuvant to facilitate sputum induction. Therefore the aim of this study was to compare chest physiotherapy and a combination of chest physiotherapy with rebound exercise using heart, respiratory, blood pressure and oxygen saturation rates, sputum volume and AFB yield in HIV patients co-infected with TB.

\section{METHODOLOGY}

\section{Population and sampling}

This single-blind randomised pre-test, post-test study was conducted from January 2012 to June 2013. Outpatients attending the local HIV clinic at King Edward VIII public hospital in KwaZuluNatal presenting with signs and symptoms of pulmonary tuberculosis (PTB) requiring sputum specimens for microscopy culture were recruited. Patients were randomised equally, using a computer, to either the control group receiving chest physiotherapy, or the experimental group performing rebound exercise followed by chest physiotherapy.

\section{Inclusion and exclusion criteria}

Patients having one or more of nonproductive cough, fever, night sweats, haemoptysis and TB contact within the past 12 months confirmed by X-ray and could not induce sputum spontaneously, served as inclusion criteria. Prior to referral for physiotherapy, patients were screened by the referring medical practitioner for resting ECG and physi- cal examination in accordance with the American Heart Association guidelines for exercise activity (American College of Sports Medicine 2000). Patients with a temperature greater than $38^{\circ} \mathrm{C}$, having coronary artery disease, myocardial infarction, cardiac or abdominal surgery within the previous 6 months, history of fractures of the spine, hip, knee and ankle joints, lower limb weakness and deformities, involvement in any other exercise programme during the period of study, or potential of non-compliance with the exercise, were excluded.

\section{Ethical issues}

The design of this study conformed to the Helsinki Declaration standards and followed ethical approval by the Biomedical Ethics Committee of the University of KwaZulu-Natal (BE 269/011). Permission to use the hospital was obtained from the Secretary General of Health for KwaZulu-Natal, and written informed consent (in English and Zulu) was obtained from the patients before enrolment into the study.

\section{Interventions}

Patients assigned to the control group received 30 minutes of chest physiotherapy, and the experimental group performed 15 minutes of rebound exercise, followed by 15 minutes of chest physiotherapy each morning over three consecutive days. The chest physiotherapy group assumed six standard postural drainage positions for five minutes each, in which chest percussions, shakings and vibrations of the chest wall were done, using a Vita-Med (Rödemark, West Germany) at $50 \mathrm{~Hz}$, followed by deep-breathing, forced expirations and vigorous coughing, which conformed to international standards (McIlwaine et al. 1997).

As an additional safety precaution due to potential respiratory compromise, patients assigned to the experimental group had to complete a six-minute walk test before engaging in rebound exercise (Mac Gavin et al. 1978). Prior to exercise activity, patients practised on the mini-trampoline Model T-rebound (Rebound Dynamics, Europe, Dietikon, Switzerland) with 36 springs and a circumference of 1.25 metres fitted with a U-frame with hand bars for safety. 
Bounce frequency signalled by an electronic metronome, was set at a minimum of 60 per minute and height of $15 \mathrm{~cm}$, which does not provoke an increase in VO2 or energy expenditure (Smith and Bishop1988). Rebound exercise of 5 minute durations and resting intervals of 2 minutes were repeated three times, with patients monitored throughout the exercise for subjective fatigue, dyspnoea, respiratory distress, profuse sweating, inability to maintain the exercise intensity, or unsteady gait, which were clinical signs of maximum excursion. Following 15 minutes of rebound exercises, patients received 15 minutes of chest physiotherapy in the identical positions as the control group.

\section{Cautionary measures}

An increase of $30 \%$ from baseline (preintervention) values of systolic or diastolic blood pressure during the exercise was an indication that the patient was reaching an unstable state, and the exercise was to be stopped (Lim et al 1996). Moderate intensity of exercise was between $50 \%-60 \%$ of the age-predicted maximum heart rate set at within 10 beats of this percentage predicted maximum for safety (American College of Sports Medicine 1995).

\section{Data collection methods and instruments}

The patient's age in years, body mass measured on a balance beam scale recorded to the nearest $0.25 \mathrm{~kg}$, and height using a wall-mounted stadiometer to the nearest $0.25 \mathrm{~cm}$, were recorded as shown in Table 1. Heart rates by means of polar heart rate monitors (Polar Electro,
Oy, Finland), respiratory, blood pressure and oxygen saturation using a portable Datex-Ohmeda Cardiocap 5 tested and calibrated by a technician before use, were used to monitor readings throughout the exercise with relevant pre-and post-readings recorded (Table 2). To maximise sputum volume during and following the interventions, patients performed three forceful huffs followed by vigorous coughing, as accounting for swallowed sputum and an admixture of saliva can be a limiting factor when collecting sputum (Lannefors and Wollmer 1992). Total sputum coughed during the activity was collected in a calibrated vial and sent to the laboratory for decontamination, centrifugation, and acid fast bacilli (AFB) assay using the Ziehl-Neelsen staining with seeding onto Löwenstein-Jensen solid culture medium.

\section{Statistical analysis}

Paired t-tests were used to analyse the difference between pre-and post-values for each parameter. Statistical Package for Social Sciences version 21 for Windows (SPSS Inc., Chicago, Illinois) was used to conduct all statistical analyses with significance set at $\mathrm{p}<0.05$. All data are presented as mean $(95 \% \mathrm{CI})$ or mean \pm standard deviation.

\section{RESULTS}

The flow diagram (Fig 1) shows that 63 patients satisfied the inclusion criteria during the period of study. Three patients refused to sign the consent form, and two did not co-operate with instructions, resulting in 58 for randomisation into two equal groups. One patient did

Table 1: Baseline demographics of patients

\begin{tabular}{|l|l|l|l|}
\hline Characteristics & $\begin{array}{l}\text { Chest } \\
\text { Physiotherapy }\end{array}$ & $\begin{array}{l}\text { Rebound } \\
\text { Exercise + Chest } \\
\text { physiotherapy } \\
(\mathrm{N}=28) \\
\text { Mean }\end{array}$ & P-value \\
& $\begin{array}{l}\mathrm{N}=29) \\
\text { Mean }\end{array}$ & $\begin{array}{l}21(75 \%) \\
7(25 \%)\end{array}$ & \\
\hline Gender: & $21(72 \%)$ & $21.4 \pm 11.1(22-49)$ & 0.73 \\
Male (\%) & $8(28 \%)$ & $69.7 \pm 13.6$ & 0.94 \\
Female (\%) & $20.5 \pm 9.8(18-44)$ & 0.69 \\
\hline Age (years) (Range) & $70.5 \pm 11.2$ & $168.9 \pm 10.8$ & \\
\hline Weight $(\mathrm{kg}) \pm$ SD & $171.6 \pm 11.2$ & & \\
\hline Height (cm) \pm SD & & & \\
\hline
\end{tabular}

not complete rebound exercises because of nausea, resulting in 18 patients for analysis in the experimental group. The baseline information is described in Table 1, with no significant differences between the groups and all pre-and postvalues recorded as mean, and reflected in Table 2. Sputum volumes did not show a significant difference between pre-and post-readings in the control group receiving chest physiotherapy $(p=0.05)$ but significance was noted in the rebound exercise with chest physiotherapy group $(\mathrm{p}=0.03)$ and also when comparing the difference between chest physiotherapy to the rebound exercise with chest physiotherapy group $(\mathrm{p}=0.04)$. The respiratory rates in the chest physiotherapy group showed no significance between pre- and post-values, but there was significance in the combination of rebound exercise with chest physiotherapy $(p=0.03)$ and on comparison of the difference between chest physiotherapy only with rebound exercise and chest physiotherapy $(p=0.02)$. Blood pressure showed no significant difference preand post- readings in the group receiving chest physiotherapy, but significance was noted pre- and post-readings in the rebound with chest physiotherapy group $(p=0.04)$. Oxygen saturation showed no significant differences in both groups. Acid fast bacilli sensitivity was higher in the rebound with chest physiotherapy group than the group receiving chest physiotherapy $(54 \%$ and $38 \%$ respectively) (Table 2).

\section{DISCUSSION}

This is the first randomised study to determine sputum volumes, comparing chest physiotherapy to a combination with rebound exercises for HIV patients co-infected with TB receiving HAART. This study supports other studies for the use of chest physiotherapy for obtaining sputum from TB and HIV patients (Pinto and Bammann 2007; Zar et al. 2000) and using exercises to aid rather than substitute for chest physiotherapy (Salh et al. 1989) as a significant volume of sputum was produced in combination with rebound exercise. The results from this study refutes suggestions that chest physiotherapy is only beneficial in lung pathologies with large volumes of spu- 
tum (McCool and Rosen 2006) or the use of airway clearance techniques be discontinued as part of the sputum induction procedure (Elkins 2005), because chest physiotherapy did produce sputum for microscopy culture. The $97 \%(n=18)$ compliance with rebound exercises shows that this exercise is suitable for HIV and TB co-infected patients, and that these patients are physically active, as their healthy peers supporting Hall and DeCharmoy (2002) that even if exercise capacity is reduced with PTB, there is no impact on function. The significant sputum produced with rebound exercises may relate to the exercise facilitating strength, and function of the respiratory system which influences pulmonary pressures, volume, breathing depth and frequency (Clanton and Diaz 1989). Physiologically inspiratory muscles including the diaphragm are morphologically and functionally skeletal muscle, and patients with respiratory compromised elastic lung tissue will have hyperinflation with shortening and flatting of the diaphragm, resulting in altered length and decreased force production (Enrich et al. 2006). Rebound exercise requiring rhythmical bouncing on the elastic surface influenced by gravity and springs enhanced diaphragm movements and lung tissue into repetitive "wave-like movements" facilitating breathing excursions and mucus clearance by hyperventilation, leading to high air flow and mechanical squeezing. This increases mucus clearance by mimicking the active cycle of breathing techniques and the natural work of breathing, which clears a significant volume of secretions from the bronchial tree, improving broncho-pulmonary secretions and lung function (Márquez et al. 2010; Pryor and Webber 1999). In addition to this, vital capacity decreases when a subject shifts from an upright to a supine position, which restricts diaphragm movements due to upward displacement of the viscera, and may be the reason for no significant changes in respiratory rates and sputum volumes as noted in patients receiving only chest physiotherapy (Goldsmith 1991). Respiratory rates increased significantly in the rebound exercise and chest physiotherapy group, as some patients may have bounced more than the prescribed minimum of 60 per minute, resulting in an increase in step frequency which increased respiratory rates significantly with no significant increases on heart rates, which are similar findings in the study by Weston et al, (2001). These findings support the use of rebound exercise, as generally during treadmill exercises an increase in heart rates has a linear increase in VO2 (Weston et al. 2001) which may be reduced in respiratory compromised patients having HIV and TB co-infection. Chest physiotherapy positions do not produce any significant effects on heart rates (Moe et al. 1990) which was also noted in this study. The hypertensive readings post-rebound exercise could be related to positive and negative lower body position changes, which alter central and thoracic blood volumes, causing changes in central venous pressure and mean values of systolic blood pressure. Systolic blood pressure also increases with exercise duration, peaking at 12 minutes at a heart rate of 160 beats/min and diastolic pressure remaining fairly constant (Coast et al. 1998; Olsen et al 2002). During chest physiotherapy, blood pressure may be a blunted baroreflex response, or the increase in central blood volume was not sufficient to stimulate high-pressure baroreceptors (Naylor et al. 2005) correlating with this study, as the chest physiotherapy group showed no significant changes in blood pressure rates.

\section{CONCLUSION}

This study supports rebound exercise as a potential adjuvant with chest physiotherapy for sputum induction in patients coinfected with TB and HIV on HAART. This was because a significant amount of sputum was produced, and there was an increase in the AFB positive yield. The additional benefit of less time in direct contact with infected patients by physiotherapists could reduce nosocomial TB infections and minimise direct interaction with an already overburdened work force. However, as the combination of rebound exercise with chest physiotherapy is a cough-generating and respiratory facilitated procedure, biosafety issue must be implemented, and rebound exercise should be used with caution due to increased respiratory and blood pressure rates.

\section{LIMITATIONS OF THE STUDY}

Interpretation of the data in this study has limitations as the inclusion criteria did not take into account the patients' severity of TB infection when being assigned to the groups, and although patients were encouraged to cough out all sputum produced, there is a possibility that some sputum could have been swallowed or saliva could have been included in the sample, thereby influencing sputum volumes.

\section{ACKNOWLEDGEMENTS}

The researchers extend their gratitude to the medical staff of the ARV unit, physiotherapists of King Edward VIII hospital for their assistance, and the ICU technician Mr P Singh, for his supervision and use of the portable Datex-Ohmeda Cardiocap 5.

\section{REFERENCES}

American College of Sports Medicine 2000 Guidelines for exercise testing and prescription 6th edn. Williams \& Wilkins, Philadelphia

American College of Sports Medicine 1995 General principles of exercise prescription 5th edn. Williams and Wilkins, Baltimore (MD)

Carter AE 1988 The new miracles of rebound exercise pp19-51. ReboundAir Inc: Nature Distributors, New York

Clanton TL, Dias PT 1989 Clinical Assessment of the respiratory muscles. Physical Therapy 75: 983-995

Coast RJ, Kroy JA, Akers FM, Dahl T 1998 Effects of lower body pressure changes on pulmonary function. Medicine \& Science in Sports and Exercise 30 (7): 1035-1040

Elkins MR, Lane T, Goldberg H, Pagliuso J, Garske LA, Hector E, Marchetto L, Alison J A, Bye PTP 2005 Effect of airway clearance techniques on the efficacy of the sputum induction procedure. European Respiratory Journal 26: 904-908

Enrich SJ, Unnithan VB, Heward C, Withnall L, Davies DH 2006 Effect of high-intensity inspiratory muscle training on lung volumes, diaphragm thickness and exercise capacity in subjects who are healthy. Physical Therapy 86 (3): 345-354

Goldsmith SR 1991 Impaired suppression of plasma nor-epinephrine during head-down tilt in patients with congestive heart failure. American Heart Journal 122: $104-107$ 
Hall M, DeCharmoy S 2002 Exercise capacity in pulmonary tuberculosis. South African Journal of Physiotherapy 58(2): 9-14

Keyser RE, Peralta L, Cade WT, Miller S, Anixt J 2000 Functional aerobic impairment in adolescents seropositive for HIV: A quasi-experimental analysis. Archives of Physical Medicine and Rehabilitation 81(11): 1479-1484

Lannefors L, Wollmer P 1992 Mucus clearance with three chest physiotherapy regimes in cystic fibrosis: a comparison between postural drainage, PEP and physical exercise. European Respiratory Journal 5:748-753

Lim PO, MacFayden RJ, Clarkson PB, MacDonald TM 1996 Impaired exercise tolerance in hypertensive patients. Annals of International Medicine 124: 41-55

Mac Lean D, Drummond G, Macpherson C, McLaren G, Prescott R 1989 Maximum expiratory airflow during chest physiotherapy on ventilated patients before and after the application of an abdominal binder. Intensive Care Medicine 15(6): 396-399

McCool FD, Rosen MJ 2006 Non-pharmacologic airway clearance therapies. Chest 129: 250S-259S

Maharaj SS, Jeena PM 2008 Cycle ergometer and rebound exercises with chest physiotherapy - a useful adjunct for sputum expectoration in mild to moderately symptomatic HIV- infected children. South African Journal of Physiotherapy 64(3) 37-42

Márquez G, Aguado X, Alegre LM, Lago A, Acero RM 2010 The trampoline after effect: the motor and sensory modulation associated with jumping on an elastic surface. Experimental Brain Research 204: $575-584$

McGavin CR, Artvinli H, Naoe H, McHardy GJ 1978 Dyspnoea, disability and walking distance: comparison of estimates of exercise performance in respiratory disease. British Medical Journal 2: 241-243
McIlwaine PM, Wong LT, Peacock J 1997 Long term comparative trials of conventional postural drainage versus positive expiratory pressure in cystic fibrosis. The Journal of Pediatrics 131(4): 571-573

Moe GW, Canep-Anson R, Howard RJ and Armstrong PW 1990 Response of atrial natriuretic factor to postural change in patients with heart failure versus subjects with normal hemodynamics. Journal of the American College of Cardiology 16 : 599-606

Naidoo S, Jinabhai CC 2006 TB in health care workers in Kwa Zulu-Natal, South Africa. International Journal of Tuberculosis and Lung Disease 10: 676-682

Narita M, Ashkin D, Hollender ES, Pitchenik AE 1998 Paradoxical worsening of tuberculosis following antiretroviral therapy in patients with AIDS. American Journal of Respiratory Critical Care Medicine 158: 157- 161

Naylor JM, Chow CM, McLean AS, Heard R and Avolio A 2005 Cardiovascular responses to shortterm head down positioning in healthy young and older adults. Physiotherapy Research International 10: 44-59

Olsen R, Amlie A, Omvik P 2002 Blood pressure monitoring. Journal of Applied Physiology 7: 149156

Olseni L, Wolmer P 1990 Muco-ciliary clearance in healthy men at rest and during exercise. Clinical Physiology 10:381-387

Pinto VS, Bammann RH 2007 Chest physiotherapy for collecting sputum samples from HIV-positive patients suspected of having tuberculosis. International Journal of Tuberculosis and Lung Disease 11(12):1302-1307

Pryor JA, Webber BA 1999 Physiotherapy for respiratory and cardiac problems, pp. 140-147. London, Churchill Livingstone, London

Salh W, Bolton D, Dodd M, Webb AK 1989 Effect of exercise and Physiotherapy in aiding sputum expectoration in adults with cystic fibrosis. Thorax 44:1006-1008

Smit E, Crespo CJ, Semba RD, Jaworowicz D, Vlahov D, Ricketts EP, Ramirez-Marrero FA, Tang MA 2006 Physical activity in a cohort of HIVpositive and HIV-negative injection drug users. AIDS Care1 8(8): 1040-1045

Smith JF, Bishop PA 1988 Rebounding exercise: are the training effects sufficient for cardiorespiratory fitness? Sports Med 5: 6-10

Stringer WW 1999 HIV and aerobic exercise Sports Medicine 28(6): 389-395

Weston AR, Khan A, Mars M 2001 Does heart rate adequately reflect exercise intensity during minitrampoline exercise? South African Journal of Sports Medicine: 9-13

Zach M, Oberwaldner B, Hausler F 1982 Cystic fibrosis: Physical exercise versus chest physiotherapy. Archives of Disease in Childhood 57: 587 - 589

Zafiropoulos B, Alisson JA, Mc Carren B 2004 Physiological responses to the early mobilization of the intubated ventilated abdominal surgery patient. Australian Journal of Physiotherapy 50(2): 95-100.

Zar HJ, Tannenbaum E, Apolles P, Roux P, Hanslo D, Hussey G 2000 Sputum induction for the diagnosis of pulmonary tuberculosis in infants and young children in an urban setting in South Africa. Archives of Disease in Childhood 82: 305-308

Zumla A, Malon P, Henderson J, Grange JM 2000 Impact of HIV infection on tuberculosis. Postgraduate Medical Journal 76: 259-268 